\title{
Resveratrol neuroprotection in a chronic mouse model of multiple sclerosis
}

\author{
Zoe Fonseca-Kelly ${ }^{\dagger}$, Mayssa Nassrallah ${ }^{\dagger}$, Jorge Uribe, Reas S. Khan, Kimberly Dine, Mahasweta Dutt and \\ Kenneth S. Shindler*
}

Department of Ophthalmology, F.M. Kirby Center for Molecular Ophthalmology, University of Pennsylvania Scheie Eye Institute, Philadelphia, PA, USA

\author{
Edited by: \\ Hana Leiba, Kaplan Medical Center, \\ Israel \\ Reviewed by: \\ Patrick Yu Wai Man, Institute of \\ Human Genetics - Newcastle \\ University, UK \\ Prem Subramanian, Johns Hopkins \\ University School of Medicine, USA \\ Beau B Bruce, Emory University, USA \\ *Correspondence: \\ Kenneth S. Shindler, Department of \\ Ophthalmology, FM. Kirby Center for \\ Molecular Ophthalmology, University \\ of Pennsylvania Scheie Eye Institute, \\ Stellar-Chance Laboratories, 3rd Floor, \\ 422 Curie Blvd, Philadelphia, PA \\ 19104, USA. \\ e-mail: kenneth.shindler@ \\ uphs.upenn.edu \\ ' Zoe Fonseca-Kelly and Mayssa \\ Nassrallah have contributed equally to \\ this work.
}

Resveratrol is a naturally occurring polyphenol that activates SIRT1, an NAD-dependent deacetylase. SRT501, a pharmaceutical formulation of resveratrol with enhanced systemic absorption, prevents neuronal loss without suppressing inflammation in mice with relapsing experimental autoimmune encephalomyelitis (EAE), a model of multiple sclerosis (MS). In contrast, resveratrol has been reported to suppress inflammation in chronic EAE, although neuroprotective effects were not evaluated. The current studies examine potential neuroprotective and immunomodulatory effects of resveratrol in chronic EAE induced by immunization with myelin oligodendroglial glycoprotein peptide in C57/BI6 mice. Effects of two distinct formulations of resveratrol administered daily orally were compared. Resveratrol delayed the onset of EAE compared to vehicle-treated EAE mice, but did not prevent or alter the phenotype of inflammation in spinal cords or optic nerves. Significant neuroprotective effects were observed, with higher numbers of retinal ganglion cells found in eyes of resveratrol-treated EAE mice with optic nerve inflammation. Results demonstrate that resveratrol prevents neuronal loss in this chronic demyelinating disease model, similar to its effects in relapsing EAE. Differences in immunosuppression compared with prior studies suggest that immunomodulatory effects may be limited and may depend on specific immunization parameters or timing of treatment. Importantly, neuroprotective effects can occur without immunosuppression, suggesting a potential additive benefit of resveratrol in combination with anti-inflammatory therapies for MS.

Keywords: optic neuritis, multiple sclerosis, EAE, resveratrol, SIRT1, neuroprotection

\section{INTRODUCTION}

Multiple sclerosis (MS) is an inflammatory demyelinating disease of the central nervous system (CNS) that can present with a variable course of neurologic disability (Noseworthy et al., 2000). Relapsing-remitting MS, the most common form of the disease, is marked by intermittent episodes of focal neurologic dysfunction that recovers as acute inflammation resolves. However, recovery is often not complete, and it is now recognized that a significant amount of neurodegeneration also occurs in MS and correlates with accumulation of permanent neurologic deficits (Losseff et al., 1996a,b; Trapp et al., 1998; Fisher et al., 2006). Other MS patients follow a primary or secondary progressive disease course marked by a slow neurologic decline due to axonal damage and loss of neurons without discrete episodes of inflammation and demyelination (Noseworthy et al., 2000).

In both MS, and its animal model [experimental autoimmune encephalomyelitis (EAE)], autoreactive T cells against myelin antigens play a critical role in disease pathogenesis (Weiner et al., 1984; Noseworthy et al., 2000; Lock et al., 2002; Gran et al., 2004). The handful of MS medications that are available are all designed to modulate the inflammatory component of the disease, but most have limited effects on neurodegeneration and long-term neurologic disability (Johnson et al., 1995; Jacobs et al., 1996; Hickman et al., 2003; Parry et al., 2003). Resveratrol, a polyphenolic compound found in red grapes and other fruits, has numerous potentially beneficial effects including cardioprotection, antioncogenesis, and anti-oxidant, as well as both anti-inflammatory and neuroprotective effects in some systems that suggest it is a good candidate therapy for MS (De La Lastra and Villegas, 2007).

We demonstrated that a pharmaceutical formulation of resveratrol (SRT501), with enhanced absorption that retains its normal activity, prevents neuronal damage, and associated long-term neurologic dysfunction in a relapsing-remitting EAE mouse model (Shindler et al., 2007, 2010). The mechanism of these neuroprotective effects involves activation of SIRT1, an NAD-dependent deacetylase involved in cell stress responses that has previously been shown to be activated by resveratrol (Milne et al., 2007). Interestingly, neither SRT501 nor other SIRT1 activating compounds modulate inflammation in relapsing-remitting EAE (Shindler et al., 2007, 2010), suggesting resveratrol has the potential to complement current immunomodulatory MS therapies that fail to prevent neurodegeneration.

In contrast to relapsing-remitting EAE effects, resveratrol reportedly shows some immunosuppressive and immunomodulatory effects in a chronic mouse EAE model (Singh et al., 2007). However, the immunomodulatory effects were modest, and neuroprotective effects were not evaluated. In addition, a different purified unmodulated resveratrol formulation was used in 
these studies. Here we examine the potential neuroprotective and immunomodulatory effects of purified resveratrol and SRT501 in chronic EAE.

\section{MATERIALS AND METHODS EXPERIMENTAL ANIMALS}

Six week old female C57/Bl6 mice were purchased from the Jackson Laboratory (Bar Harbor, ME, USA). Housing and treatment of animals conformed to the Institutional Animal Care and Use Committee Guidelines and review at the University of Pennsylvania.

\section{INDUCTION AND SCORING OF EAE}

Experimental autoimmune encephalomyelitis was induced as in our prior studies (Quinn et al., 2011). Briefly, mice were anesthetized with $0.2 \mathrm{ml}$ solution containing $10 \mathrm{mg} / \mathrm{ml}$ ketamine (Sigma, St. Louis, MO, USA) and $1 \mathrm{mg} / \mathrm{ml}$ xylazine (Sigma) i.p. Mice were immunized with $300 \mu \mathrm{g}$ myelin oligodendroglial glycoprotein (MOG) peptide 35-55 (Invitrogen, Carlsbad, CA, USA) emulsified in complete Freund's adjuvant (CFA; Difco, Detroit, MI, USA) containing $2.5 \mathrm{mg} / \mathrm{ml}$ mycobacterium tuberculosis (Difco), divided into two doses injected subcutaneously at two separate sites on the back. Control mice were injected with an equal volume of phosphate buffered saline (PBS) and CFA. EAE and control mice received $200 \mathrm{ng}$ pertussis toxin (List Biological, Campbell, CA, USA) in $0.1 \mathrm{ml}$ PBS by i.p. injection on the day of immunization (day 0) and again on day 2. Clinical EAE was scored daily by a blinded observer with a previously used five point scale (Shindler et al., 2007, 2010; Quinn et al., 2011): no disease $=0$; partial tail paralysis $=0.5$; tail paralysis or waddling gait $=1.0$; partial tail paralysis and waddling gait $=1.5$; tail paralysis and waddling gait $=2.0$; partial limb paralysis $=2.5$; paralysis of one limb $=3.0$; paralysis of one limb and partial paralysis of another $=3.5$; paralysis of two limbs $=4.0$; moribund state $=4.5$; death $=5.0$.

\section{RESVERATROL TREATMENT}

The SRT501 (Sirtris, a GSK Company, Cambridge, MA, USA) formulation of resveratrol (Milne et al., 2007; Shindler et al., 2010) was suspended in 2\% hydroxypropyl methylcellulose (Shin-Etsu Chemicals, Japan) and $0.2 \%$ dioctyl sodium sulfosuccinate (Wilson Laboratories, Mumbai, India). Purified resveratrol purchased from Sigma was suspended in PBS. Mice were treated by oral gavage once daily with $100 \mathrm{mg} / \mathrm{kg}$ Sigma resveratrol, $250 \mathrm{mg} / \mathrm{kg}$ Sigma resveratrol, $250 \mathrm{mg} / \mathrm{kg}$ SRT501 resveratrol, or PBS alone as indicated in each experiment.

\section{HISTOLOGY}

Mice were transcardially perfused with PBS followed by $4 \%$ paraformaldehyde. Spinal cords and optic nerves were removed, post-fixed in $4 \%$ paraformaldehyde, embedded in paraffin, and cut in $5 \mu \mathrm{m}$ thick sections. For routine histological analysis, sections were stained with hematoxylin and eosin $(\mathrm{H} \& \mathrm{E})$ and examined by light microscopy. The presence or absence of inflammatory cell infiltrates, and relative degree of inflammation was scored by a blinded investigator using a previously described 0-4 point scale for optic nerves (Shao et al., 2004; Shindler et al., 2006): no infiltration $=0$, mild cellular infiltration of optic nerve or optic nerve sheath $=1$, moderate infiltration $=2$, severe infiltration $=3$, massive infiltration $=4$; and $0-3$ point scale for spinal cords: no inflammation $=0$; mild inflammation $=1$, moderate inflammation $=2$, severe inflammation $=3$.

\section{RETINAL GANGLION CELL QUANTIFICATION}

Retinal ganglion cells (RGCs) were labeled and quantified as previously described (Shindler et al., 2007, 2010; Quinn et al., 2011). Briefly, RGCs were retrogradely labeled by injection of $2.5 \mu \mathrm{l}$ of $1.25 \%$ hydroxystilbamidine (Fluorogold; Invitrogen) into the superior colliculi 1 week prior to EAE induction. Following sacrifice, isolated, fixed retinas were whole-mounted on glass slides and viewed by fluorescent microscopy. Photographs were taken at 20X magnification in 12 standard fields: $1 / 6,3 / 6$, and $5 / 6$ of the retinal radius from the center of the retina in each retinal quadrant, and the number of RGCs was counted using Image-Pro Plus 6.0 (Media Cybernetics, Silver Spring, MD, USA) software.

\section{PUPILLOMETRY}

Pupillary light responses were induced and recorded similar to prior studies using a Neuroptics Pupillometer (San Clemente, CA, USA) for rodents (Hussain et al., 2009; Shindler et al., 2012). A series of five flashes of light at an intensity of $4.7 \mu \mathrm{W} / \mathrm{cm}^{2}$, was used to stimulate each eye and the pupillometer's dual-camera system measures changes in pupillary diameter of both eyes. Pupillary constriction was determined by averaging the percentage change in pupil diameter following each flash of light. The highest and lowest pupillary responses were discarded as potential outliers for each eye, with the average of the remaining three responses used for statistical comparisons.

\section{OPTOKINETIC RESPONSES}

Visual function was assessed by the optokinetic tracking response (OKR) using OptoMotry software and apparatus (Cerebral Mechanics), as described previously (Prusky et al., 2004; Quinn et al., 2011). Briefly, mice in a closed chamber are observed through a camera to determine whether they are tracking a virtual cylinder of a $100 \%$ contrast grating projected at varying spatial frequencies. The highest spatial frequency at which mice track correlates with visual acuity and is recorded as cycles/degree.

\section{IMMUNOHISTOCHEMISTRY}

Spinal cord sections were stained by the avidin-biotinimmunoperoxidase technique (Vector Laboratories, Burlington, CA, USA) using 3, 3' diaminobenzidine as substrate, and antibodies against lymphocytic cell markers, as in prior studies (Shindler et al., 2011). Primary antibodies anti-CD3 mouse monoclonal (Santa Cruz Biotechnology, Santa Cruz, CA, USA) diluted 1:200 in PBS, anti-B220 Mouse monoclonal (BD Pharmingen, San Diego, CA, USA) diluted 1:200, and anti-Ibal rabbit polyclonal (Wako, Richmond, VA, USA) diluted 1:200 were used as markers for T cells, $\mathrm{B}$ cells, and macrophages/microglia respectively. Anti-mouse or rabbit IgG secondary antibodies from the Vectastain ABC Mouse Kit (Vector Laboratories) were used according to manufacturer's instructions. 


\section{FLOW CYTOMETRY}

Cells were isolated from EAE spinal cords and spleen by methods described previously (Fitzgerald et al., 2007). Briefly, following transcardial perfusion with PBS, spleens, and spinal cords were removed, spinal cords were pooled, and tissues were mechanically dissociated through a $100-\mu \mathrm{m}$ strainer and washed with PBS. Resultant pellets were fractionated on a $60 / 30 \%$ Percoll gradient by centrifugation at $300 \times g$ for $20 \mathrm{~min}$. Mononuclear cells were harvested from the interface, washed, counted, and stained for $20 \mathrm{~min}$ in the dark at $4^{\circ} \mathrm{C}$ with fluorescence-labeled antibodies specific for cell surface markers. For intracellular markers, cells were washed, fixed and permeabilized with Fix \& Perm reagents (Caltag Laboratories) then stained with fluorescence-labeled antibodies. All antibodies (CD4, clone RM4-5; CD8, clone 53-6.7; IL-17, clone TC11-18H10; IFN-gamma, clone XMG1.2) were purchased from BD Bioscience and were used at a concentration of $0.5 \mu \mathrm{g} / \mathrm{ml}$. Data were acquired on a FACSAria (BD Biosciences) and analyzed with FlowJo software (Treestar) with cells gated based on physical parameters (size and granularity).

\section{STATISTICS}

Clinical EAE scores were compared between treatment groups by ANOVA for repeated measures using GraphPad Prism 5.0 (GraphPad Software, San Diego, CA, USA). Comparisons of RGC numbers, inflammation severity, and percentages of inflammatory cell types were analyzed by one way ANOVA followed by Tukey's Multiple Comparison test using GraphPad Prism.

\section{RESULTS}

\section{RESVERATROL DELAYS EAE ONSET}

Experimental autoimmune encephalomyelitis was induced in 8 week old female C57/B16 mice by immunization with MOG peptide (Quinn et al., 2011). Mice were treated orally daily for 30 days with purified resveratrol (RSV) at 100 or $250 \mathrm{mg} / \mathrm{kg}$, doses reported previously to suppress EAE (Singh et al., 2007). Additional EAE mice were treated with $250 \mathrm{mg} / \mathrm{kg}$ SRT501 resveratrol, or with PBS alone. Mice treated with PBS developed a typical course of chronic EAE, marked by ascending paralysis beginning about 12 days after immunization, peaking several days later, and then persisting (Figure 1). Treatment with $100 \mathrm{mg} / \mathrm{kg} \mathrm{RSV}$ failed to suppress EAE, whereas both RSV and SRT501 given at $250 \mathrm{mg} / \mathrm{kg}$ delayed the onset of EAE by several days, although by $16-17$ days post-immunization EAE severity reached levels equivalent to PBS-treated mice (Figure 1).

\section{RESVERATROL DOES NOT REDUCE CNS INFLAMMATION}

Experimental autoimmune encephalomyelitis mice treated with RSV, SRT501, or mock-treated with PBS were sacrificed on day 30 post-immunization. Sections of spinal cords stained by $H \& E$ were examined for areas of inflammatory cell infiltration. Foci of inflammation were observed in the white matter of EAE spinal cords, and similar inflammation was detected in EAE spinal cords from mice treated with RSV and SRT501 (Figures 2A-D). There was no difference in the incidence of inflammation detected in RSV-, SRT501-,

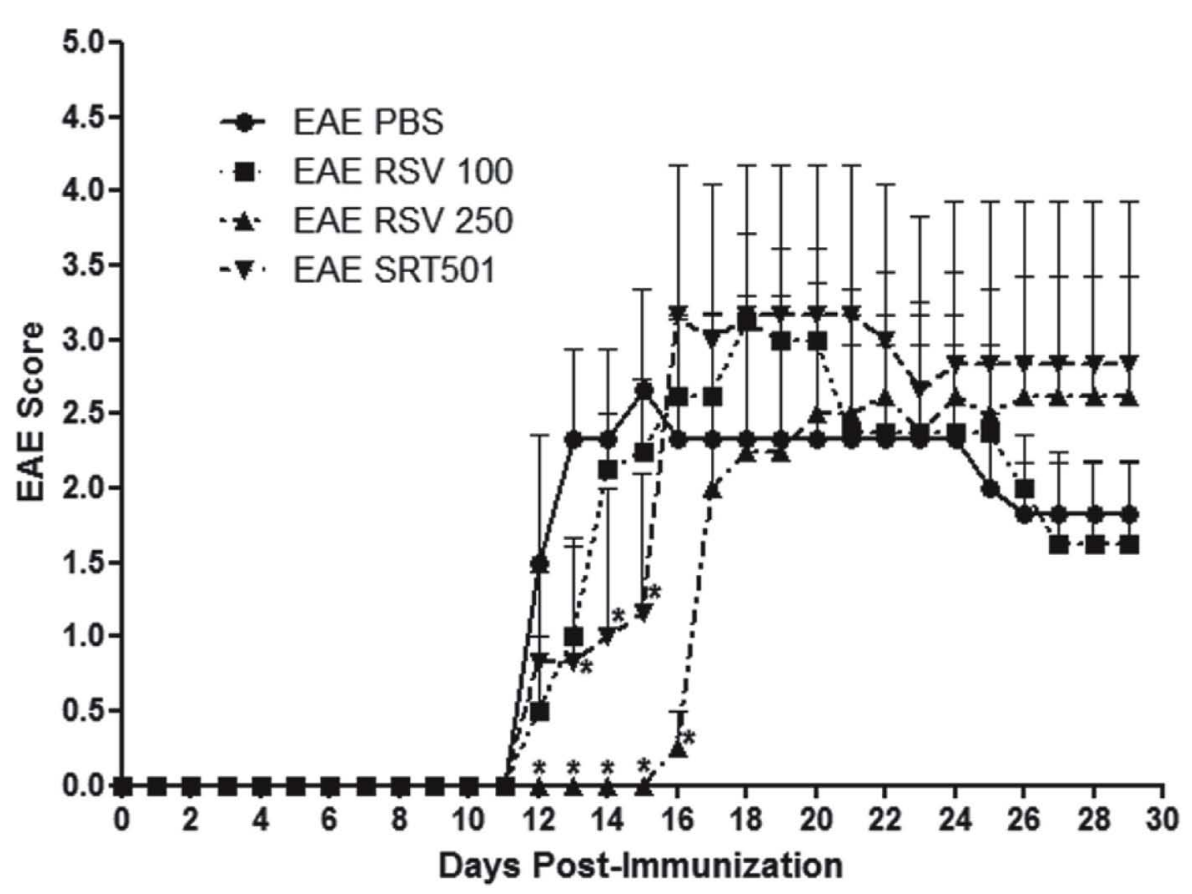

FIGURE 1 | Resveratrol delays onset of EAE. Eight week old female C57/BI6 mice were immunized with MOG on day 0 , and mice treated daily by oral gavage with PBS, $100 \mathrm{mg} / \mathrm{kg}$ RSV, $250 \mathrm{mg} / \mathrm{kg} \mathrm{RSV}$, or $250 \mathrm{mg} / \mathrm{kg}$ SRT501 ( $N=5$ mice/group). Mice were observed daily for clinical signs of $E A E$ and scored on a five point scale. No difference in EAE onset or severity was found between PBS- or $100 \mathrm{mg} / \mathrm{kg}$ RSV-treated EAE mice. Treatment with $250 \mathrm{mg} / \mathrm{kg}$ RSV or SRT501 both resulted in significant suppression of EAE at the onset of disease (days $12-16$; ${ }^{*} p<0.05$ ) as compared to EAE mice receiving PBS alone. One of two representative experiments is shown. 
A

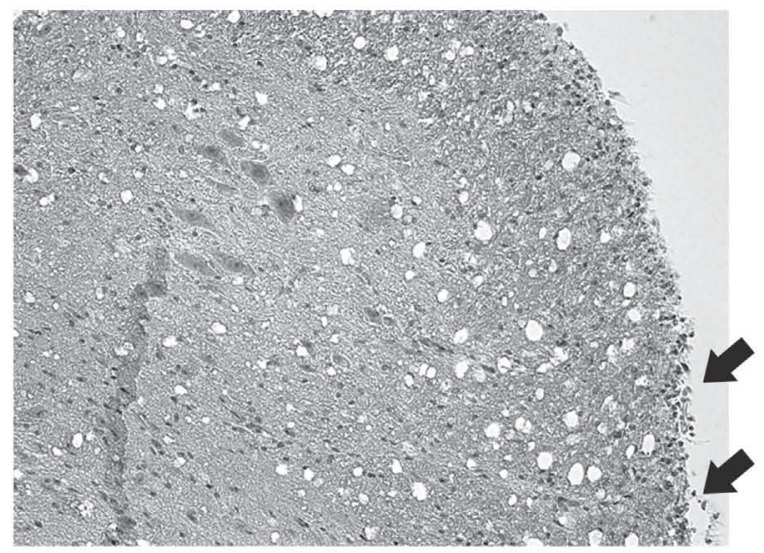

B

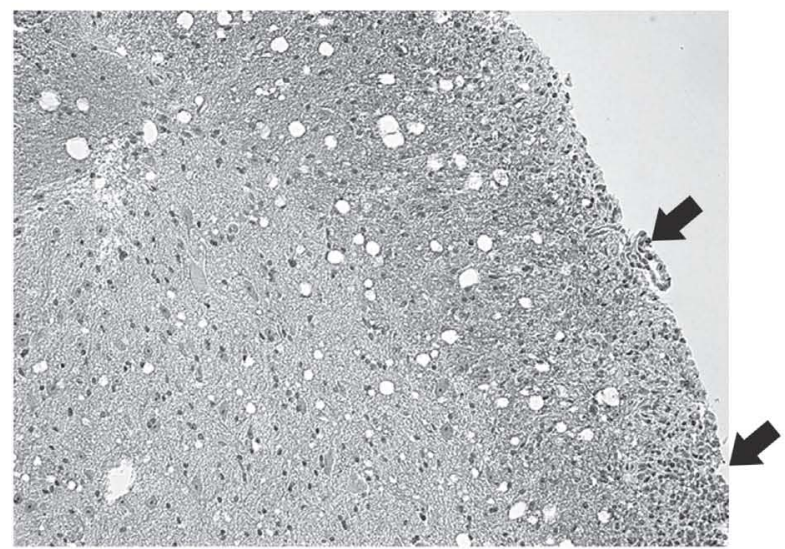

C

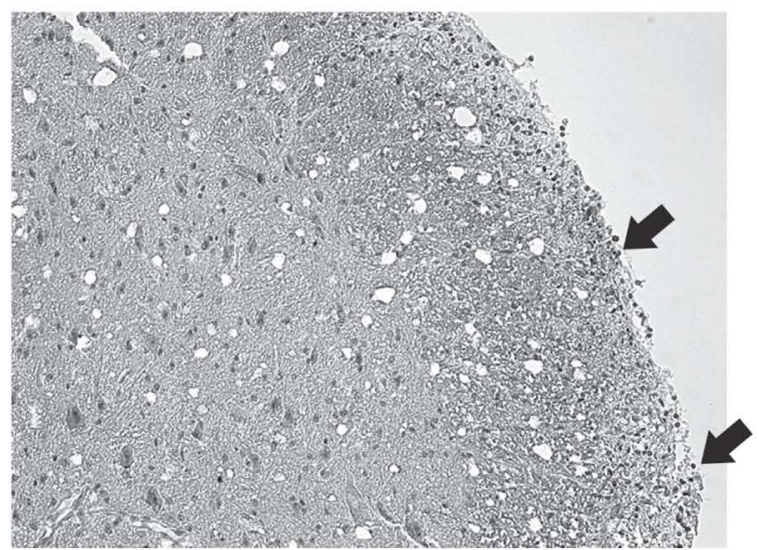

D

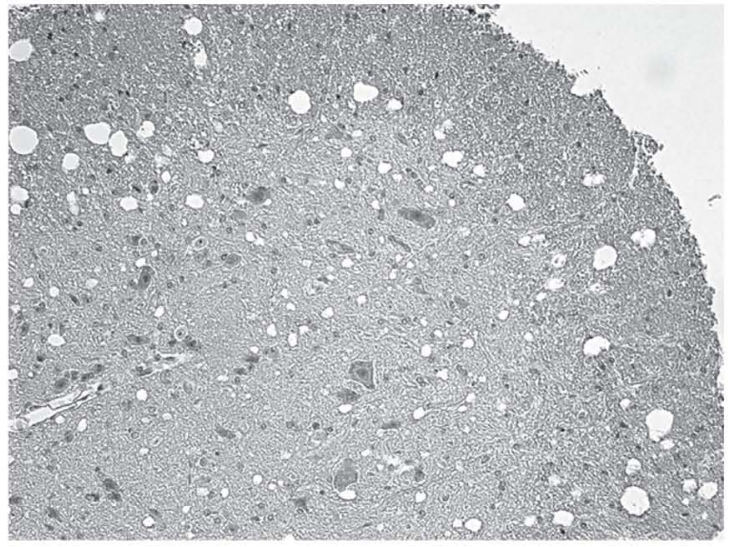

E

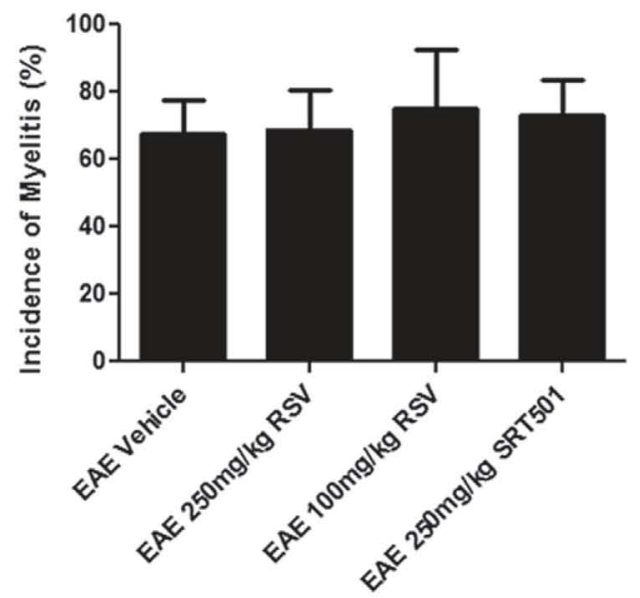

FIGURE 2 | Resveratrol does not suppress spinal cord inflammation. Spinal cord sections from EAE mice were stained by H\&E. (A) Spinal cord from an EAE mouse treated with PBS alone shows foci of increased cellularity in spinal cord white matter characteristic of inflammatory cell infiltrates (arrows). Similar inflammation (arrows) is seen in spinal cords from EAE mice treated with $250 \mathrm{mg} / \mathrm{kg}$ RSV (B) and $250 \mathrm{mg} / \mathrm{kg}$ SRT501 (C). (D) Spinal cord from a control, non-EAE mouse demonstrates the normal cellularity without inflammatory cell infiltration. (E) The percentage of spinal cord sections containing inflammatory cell infiltrates did not differ between PBS-, RSV-, or SRT501-treated EAE mice. Photos shown at original magnification $\times 20$ (A-D). and PBS-treated mouse spinal cords (Figure 2E). The average severity of spinal cord inflammation scored on a relative scale was also not different between PBS-treated (1.24 \pm 0.44 , $N=21), 100 \mathrm{mg} / \mathrm{kg}$ RSV-treated $(1.40 \pm 0.52, N=10), 250 \mathrm{mg} / \mathrm{kg}$ RSV-treated $(1.27 \pm 0.47, N=11)$, or $250 \mathrm{mg} / \mathrm{kg}$ SRT501-treated $(1.36 \pm 0.50, N=11)$ EAE mice.
Optic neuritis, inflammatory demyelination of the optic nerve, occurs frequently in MS (Arnold, 2005) and in almost all chronic EAE mice (Shao et al., 2004; Quinn et al., 2011). To further examine effects of resveratrol on EAE, optic nerve sections were examined. H\&E staining demonstrated inflammatory cell infiltrates in optic nerves from RSV-, SRT501-, and PBS-treated EAE mice, 
with no significant difference in the incidence of optic neuritis between treated and mock-treated mice (Figure 3). The severity of optic nerve inflammation, scored on a relative four-point scale (Shindler et al., 2010; Quinn et al., 2011), also showed no suppression of inflammation by RSV or SRT501, as differences in the degree of inflammation present in optic nerves from PBS-treated $(1.33 \pm 0.49, N=18), 100 \mathrm{mg} / \mathrm{kg}$ RSV-treated $(1.85 \pm 0.89, N=7), 250 \mathrm{mg} / \mathrm{kg}$ RSV-treated $(1.14 \pm 0.38, N=7)$, or $250 \mathrm{mg} / \mathrm{kg}$ SRT501-treated $(1.56 \pm 0.53, N=9)$ EAE mice were not statistically significant $(p=0.10)$.

\section{RESVERATROL REDUCES NEURONAL LOSS IN EAE MICE}

Experimental autoimmune encephalomyelitis optic neuritis leads to significant loss of RGCs, the neurons that form the optic nerve, and associated decreased visual function (Quinn et al., 2011). To examine whether resveratrol can protect neurons from damage in EAE, RGCs were retrogradely labeled with fluorogold by injection into the superior colliculi 1 week prior to EAE induction (Shindler et al., 2007, 2010; Quinn et al., 2011). Mice were treated daily with RSV, SRT501, or mock-treated with PBS following immunization, and control non-EAE mice were sham immunized without peptide. RGC function was assessed by measuring pupillary light responses using pupillometry (Hussain et al., 2009), and visual acuity was estimated by OKR (Prusky et al., 2004). There was a gradual decline in pupillary responses over the course of 4 weeks, with no differences observed between treated or mocktreated EAE mice (Figure 4A). Visual function decline was also observed in all treatment groups. Both RSV and SRT501 delayed this loss of OKR responses at 2 weeks post-immunization, with visual function significantly better than in mock-treated EAE mice (Figure 4B).
Retinal ganglion cells survival was assessed 30 days postimmunization. The number of surviving RGCs was measured by counting cells in 12 standardized fields on retinal whole mounts (Shindler et al., 2007, 2010; Quinn et al., 2011). Eyes from EAE mice mock-treated with PBS had significantly fewer RGCs (335.8 \pm 28.8 RGCs/eye) than control, non-EAE mice (529.2 \pm 36.2$)$, similar to prior studies (Quinn et al., 2011). Eyes from EAE mice treated with $100 \mathrm{mg} / \mathrm{kg}$ RSV (458.8 \pm 25.3 ), $250 \mathrm{mg} / \mathrm{kg}$ RSV (529.2 \pm 82.0 ), and $250 \mathrm{mg} / \mathrm{kg}$ SRT501 (464.6 \pm 43.0$)$ all had significantly more RGCs than eyes from mock-treated EAE mice (Figure 4C). Thus, on average, untreated EAE mouse eyes lost 193.4 (36.5\%) of their RGCs, and treatment with $100 \mathrm{mg} / \mathrm{kg}$ RSV, $250 \mathrm{mg} / \mathrm{kg}$ RSV, and $250 \mathrm{mg} / \mathrm{kg}$ SRT501 reduced the amount of RGC loss by 64, 100, and $66.5 \%$, respectively.

\section{RESVERATROL DOES NOT MODULATE PHENOTYPE OF CNS INFLAMMATION}

While results show that RSV and SRT501 both prevent neuronal loss in EAE and delay neurologic dysfunction without suppressing the gross level of inflammation that occurs in the spinal cord and optic nerve, it is possible that resveratrol may reduce neuronal damage by altering the type of inflammatory cells that enter the CNS. To assess the basic types of inflammatory cells present, sections of spinal cord from mock-, RSV-, and SRT501treated EAE mice were stained with markers of T cells (CD3), $B$ cells (B220), and macrophages/microglia (Iba1). Inflammatory lesions in EAE spinal cord contained numerous T cells, as well as macrophages, with little or no B cells present, similar to prior studies, demonstrating the ability of these antibodies to detect expected cell types (Figure 5). To quantify the relative contribution of different cell types in spinal cord infiltrates, the presence of each type
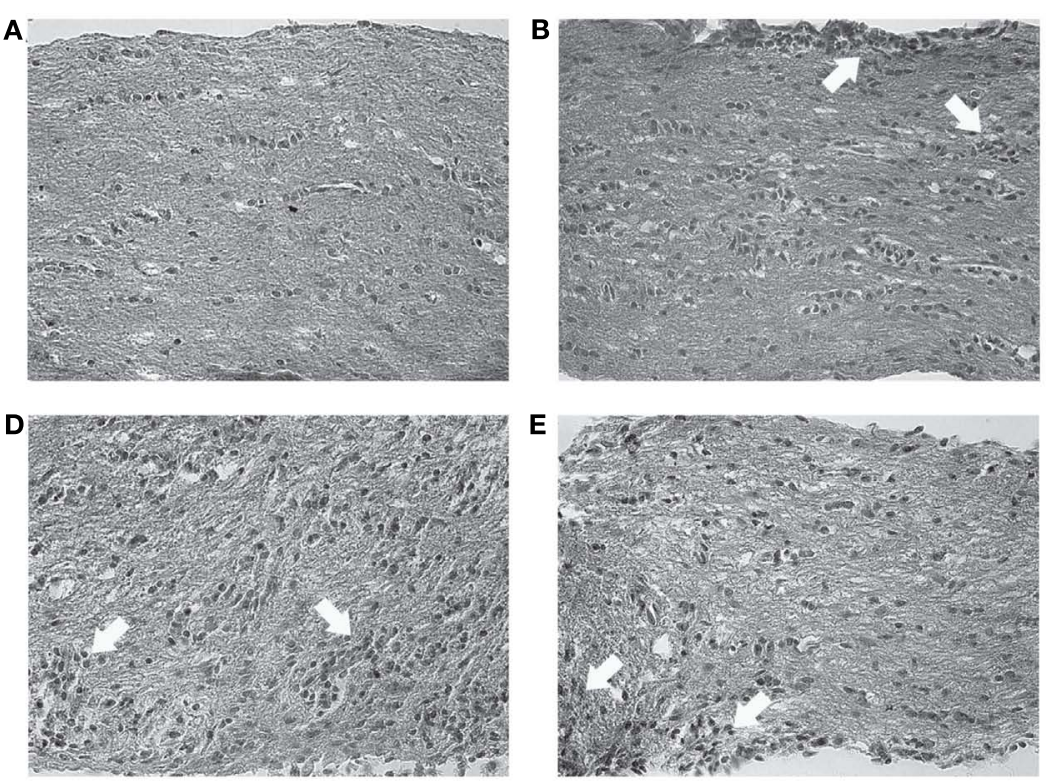

$\mathbf{E}$

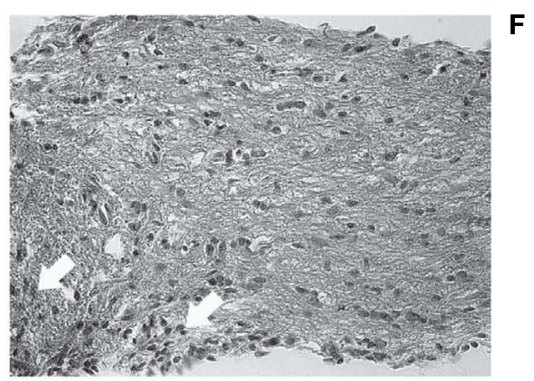

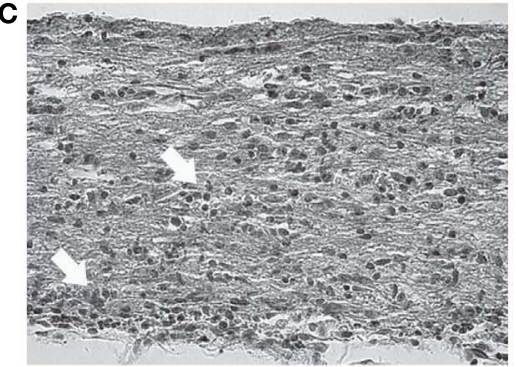

$\mathbf{F}$

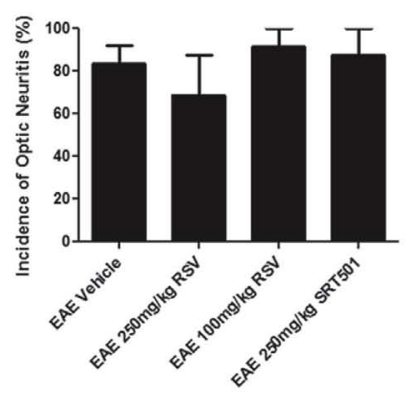

FIGURE 3 | Resveratrol does not suppress optic nerve inflammation. Optic nerve sections from EAE mice were stained by H\&E. (A) Normal cellularity of a control, non-EAE optic nerve without inflammation is shown. (B) Optic nerve from an EAE mouse treated with PBS alone shows foci of inflammatory cell infiltrates (arrows) within the optic nerve and optic nerve sheath. Similar inflammation (arrows) is seen in optic nerves from EAE mice treated with $100 \mathrm{mg} / \mathrm{kg}$ RSV (C), $250 \mathrm{mg} / \mathrm{kg}$ RSV (D), and $250 \mathrm{mg} / \mathrm{kg}$ SRT501 (E). (F) The percentage of inflamed optic nerves did not differ between PBS-, RSV-, or SRT501-treated EAE mice. Photos shown at original magnification $\times 40$ (A-E) 


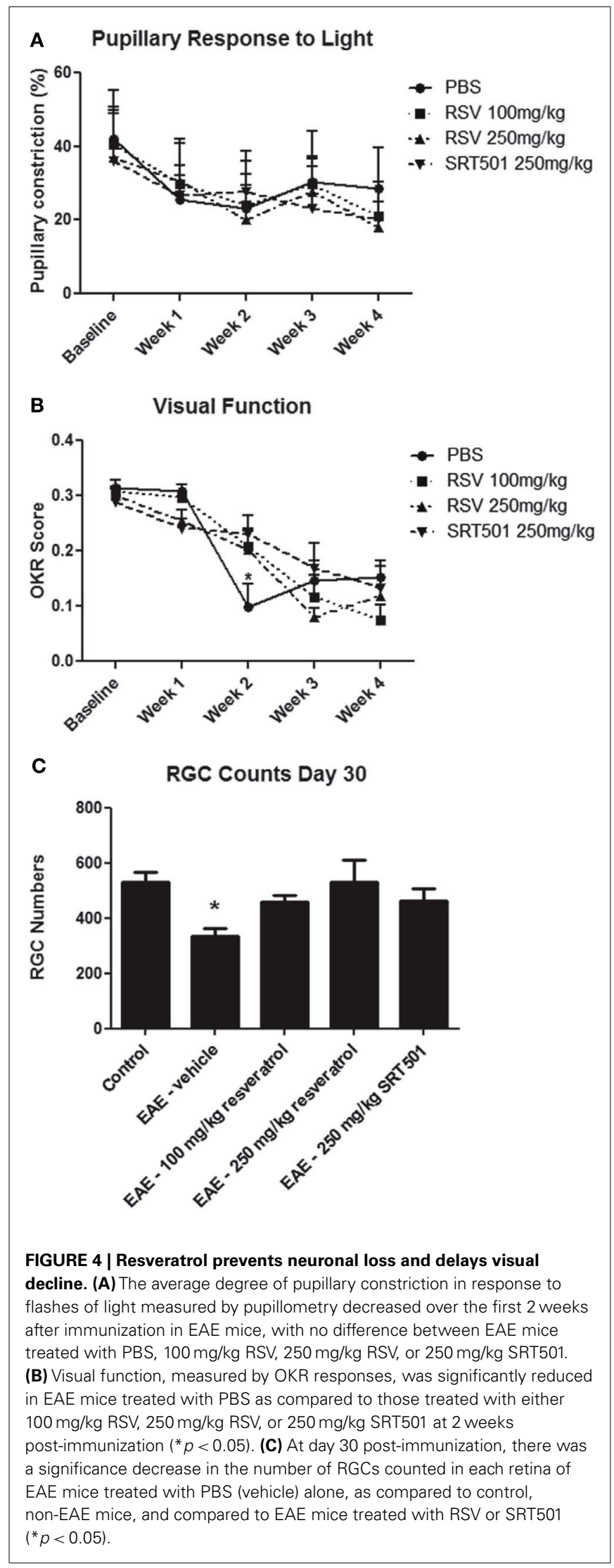

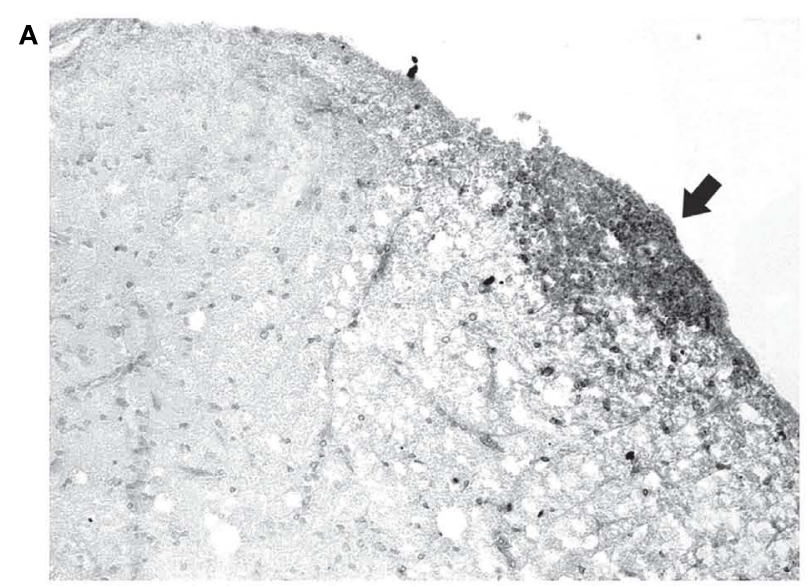

B

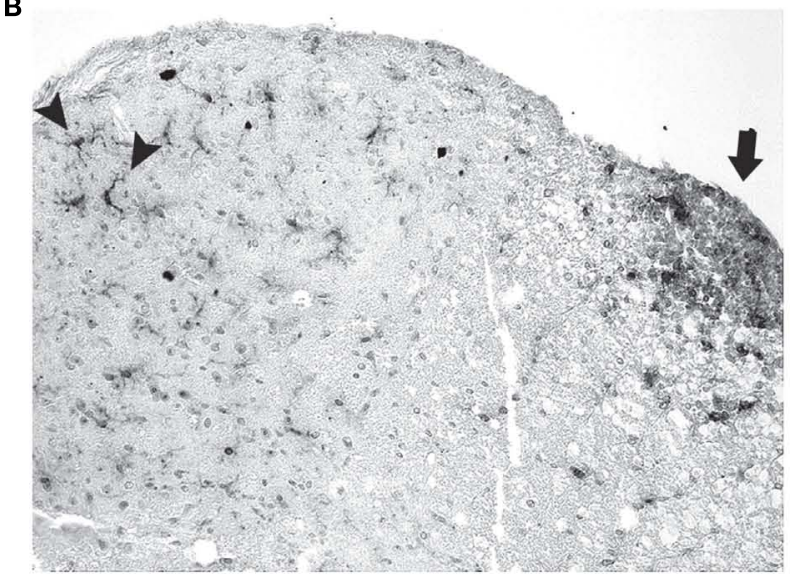

C

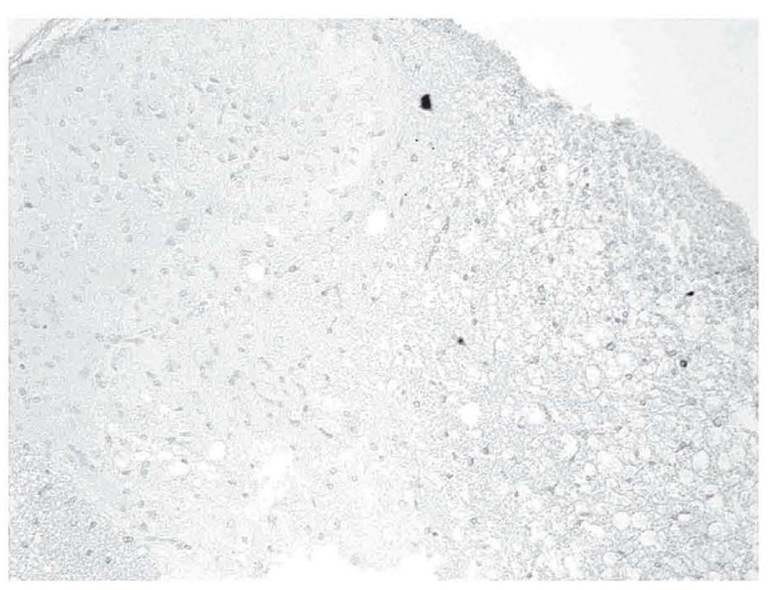

FIGURE 5 | Inflammatory cell types detected in EAE spinal cord. (A)

Section of a representative EAE spinal cord 30 days post-immunization stained with antibodies to $\mathrm{CD} 3$ shows numerous $T$ cells mainly within the white matter (arrow). (B) An adjacent section stained with Iba1 antibodies demonstrates numerous macrophages/microglia in the same inflammatory focus (arrow) in the white matter, as well as individual microglia across the spinal cord white matter and gray matter (arrowheads). (C) Adjacent section stained with B220 antibodies shows B cells do not contribute significantly to observed foci of inflammation. Photos shown at original magnification $\times 20(\mathbf{A}-\mathbf{C})$. 
of inflammatory cell, and the relative level of inflammation, was score by a blinded investigator. No significant difference in the amount of inflammation by each cell type was observed between RSV-, SRT501-, or mock-treated mice (Table 1).

To further examine the phenotype of inflammation, mononuclear cells were isolated from the spinal cords of EAE mice and analyzed by flow cytometry, and cells isolated from the spleen were analyzed to assess effects on T cells in the periphery. Results show no significant differences in the percentage of CD4+ vs. CD8+ $\mathrm{T}$ cells present in spinal cords or spleens from RSV-, SRT501-, or mock-treated EAE mice (Figure 6). As both interferon(IFN)gamma + Th1 effector T cells and IL-17+ Th17 effector T cells have been implicated as the pathogenic cells in EAE (Langrish et al., 2005; Steinman, 2008; Stromnes et al., 2008), we also examined the expression of these cytokines in CNS and peripheral T cells. The proportion of IFN-gamma and IL-17 expressing T cells was limited to less than $10 \%$ in the spleen and was only slightly higher in spinal cord. Notably, no differences were found between treated and mock-treated mice (Figure 6).

\section{DISCUSSION}

Results demonstrate that oral SRT501 resveratrol prevents neuronal damage in chronic EAE, similar to the neuroprotective effects observed previously in relapsing-remitting EAE (Shindler et al., 2010). SRT501 effects on RGCs are robust, eliminating twothirds of the RGC loss induced by EAE optic neuritis. Effects are not specific to SRT501, but are also induced by unmodified, purified resveratrol. As in the relapsing-remitting EAE model, the mechanism of these neuroprotective effects does not involve

Table 1 | Levels of lba1 + macrophages/microglia, CD3 + T cells, and B220+ $B$ cells detected in EAE spinal cords.

\begin{tabular}{lllll}
\hline Iba1 & & & & \\
EAE PBS & $0 \%(0 / 12)$ & $8 \%(1 / 12)$ & $58 \%(7 / 12)$ & $33 \%(4 / 12)$ \\
EAE RSV & $0 \%(0 / 9)$ & $11 \%(1 / 9)$ & $44 \%(4 / 9)$ & $44 \%(4 / 9)$ \\
EAE SRT501 & $0 \%(0 / 9)$ & $0 \%(0 / 9)$ & $44 \%(4 / 9)$ & $56 \%(5 / 9)$ \\
CD3 & & & & \\
& - & \pm & + & ++ \\
EAE PBS & $36 \%(4 / 11)$ & $27 \%(3 / 11)$ & $27 \%(3 / 11)$ & $9 \%(1 / 11)$ \\
EAE RSV & $44 \%(4 / 9)$ & $22 \%(2 / 9)$ & $22 \%(2 / 9)$ & $11 \%(1 / 9)$ \\
EAE SRT501 & $11 \%(5 / 9)$ & $22 \%(2 / 9)$ & $56 \%(5 / 9)$ & $11 \%(1 / 9)$ \\
B220 & & & & \\
& - & \pm & + & ++ \\
EAE PBS & $55 \%(6 / 11)$ & $45 \%(5 / 11)$ & $0 \%(0 / 11)$ & $0 \%(0 / 11)$ \\
EAE RSV & $89 \%(8 / 9)$ & $11 \%(1 / 9)$ & $0 \%(0 / 9)$ & $0 \%(0 / 9)$ \\
EAE SRT501 & $56 \%(5 / 9)$ & $44 \%(4 / 9)$ & $0 \%(0 / 9)$ & $0 \%(0 / 9)$ \\
\hline
\end{tabular}

(-), no stained cells observed; ( \pm ), equivocal staining with just a few rare positive cells present; (+), scattered definitively positive staining cells in spinal cord white matter; (++), discreet foci of positive cells. The percentage of spinal cords from EAE mice treated with PBS, $250 \mathrm{mg} / \mathrm{kg}$ RSV, or $250 \mathrm{mg} / \mathrm{kg}$ SRT501 for 30 days that demonstrated each level of staining on immunohistochemistry is shown. Numbers in parentheses indicate the number of spinal cords receiving each score/total number examined.
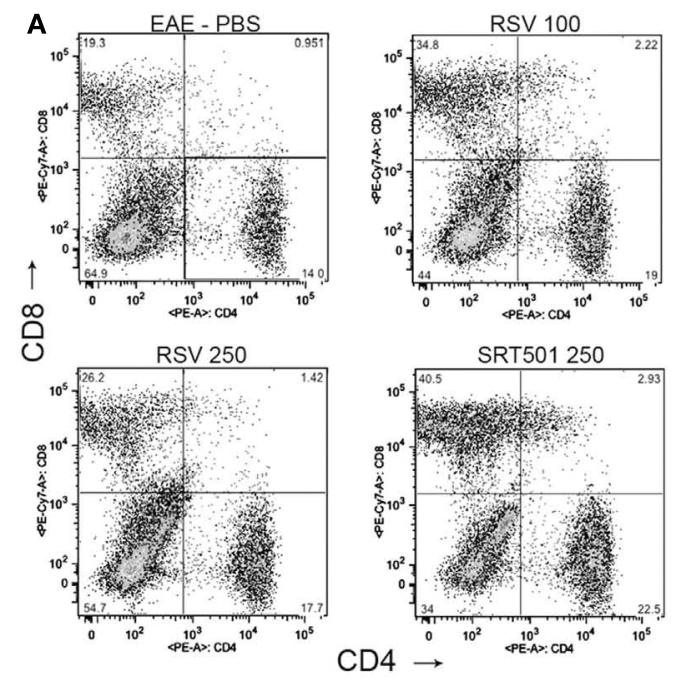

B

Spleen

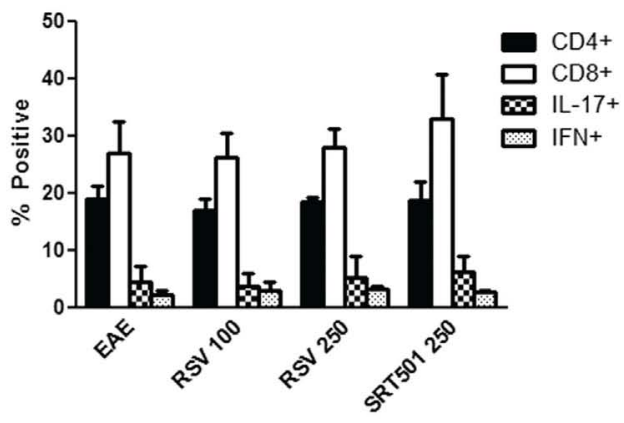

C Spinal cord

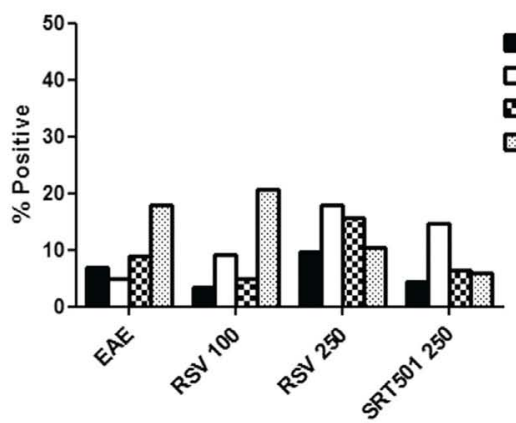

FIGURE 6 | Resveratrol does not suppress T cells in the periphery or CNS. Total cells were isolated from the spleens of individual EAE mice treated with PBS alone, $100 \mathrm{mg} / \mathrm{kg} \mathrm{RSV}, 250 \mathrm{mg} / \mathrm{kg} \mathrm{RSV}$, or 250 SRT501 daily until day 30 post-immunization. (A) Freshly isolated cells stained with antibodies to $\mathrm{CD} 4$ and $\mathrm{CD} 8$ were analyzed by flow cytometry.

Representative plot for one mouse in each treatment group shows identifiable populations of CD4+ and CD8+T cells. (B) The average percentage of $\mathrm{CD} 4+$ and $\mathrm{CD} 8+\mathrm{T}$ cells found in $\mathrm{EAE}$ spleens ( $N=4$ mice/treatment group), and the percentage of $\mathrm{CD} 4+$ cells that stained positive for IL-17 and IFN-gamma, is shown. No difference is observed between treatment groups. (C) Spinal cords from mice in each treatment group $(N=4)$ were pooled together to ensure sufficient numbers of inflammatory cells for flow cytometry. Less than $10 \%$ of spinal cord mononuclear cells from each treatment group were $C D 4+T$ cells. Within the $\mathrm{CD} 4+\mathrm{T}$ cell population, $5-15 \%$ were IL- $17+$ and $10-20 \%$ were IFN-gamma+, with too few cells present for statistical analysis. 
significant long-term suppression or modulation of inflammation, as the level of inflammation and type of cells present was not altered by resveratrol during the chronic stage of EAE examined in these studies. In addition to preventing loss of neurons, at daily doses of $250 \mathrm{mg} / \mathrm{kg}$ both resveratrol compounds significantly delay the development of neurologic dysfunction - ascending paralysis marked by EAE score, and visual function measured by OKR.

Despite observed neuroprotective effects, resveratrol's ability to prevent paralysis and vision loss is limited in this chronic autoimmune disease. Within several days of the onset of symptoms, EAE severity and visual decline worsen to levels similar to those in mock-treated EAE controls. This eventual accumulation of neurologic deficits likely is due to the chronic inflammatory nature of this model. Indeed, by 30 days post-immunization, RSV-, SRT501-, and mock-treated EAE mice all have similar levels of spinal cord and optic nerve inflammation which may be directly interfering with nerve function despite improved neuronal survival. Similar findings were observed in relapsing-remitting EAE mice treated with SRT501 (Shindler et al., 2010), where SRT501 failed to improve neurologic function during the acute inflammatory phase of disease, but in those mice SRT501 treatment did significantly improve neurologic function during the remission phase of disease, after inflammation resolved. The current results demonstrating the ability to maintain neurons up to 30 days post-immunization despite ongoing inflammation suggests that resveratrol has the potential to delay neuronal loss for longer periods of time during exposure to damaging inflammation, and may be capable of maintaining neurons while waiting for current immunomodulatory MS medications to successfully suppress active inflammation. This is particularly important for MS because permanent neurologic disability that affects many MS patients occurs secondary to neuronal loss (Losseff et al., 1996a,b). The ability of resveratrol to protect RGCs suggests the potential for improved visual outcomes following optic neuritis, where it has been well documented that RGC axonal loss correlates with vision loss (Steel and Waldock, 1998; Parisi et al., 1999; Trip et al., 2005; Costello et al., 2006; Fisher et al., 2006).

Unlike prior studies (Singh et al., 2007) that reported sustained suppression of EAE using the same 100 and $250 \mathrm{mg} / \mathrm{kg}$ doses tested here, we found only a delay in EAE development

\section{REFERENCES}

Arnold, A. C. (2005). Evolving management of optic neuritis and multiple sclerosis. Am. J. Ophthalmol. 139, 1101-1108.

Costello, F., Coupland, S., Hodge, W., Lorello, G. R., Koroluk, J., Pan, Y. I., Freedman, M. S., Zackon, D. H., and Kardon, R. H. (2006). Quantifying axonal loss after optic neuritis with optical coherence tomography. Ann. Neurol. 59, 963-969.

De La Lastra, C. A., and Villegas, I. (2007). Resveratrol as an antioxidant and pro-oxidant agent: mechanisms and clinical implications.
Biochem. Soc. Trans. 35, 1156-1160.

Fisher, J. B., Jacobs, D. A., Markowitz, C. E., Galetta, S. L., Volpe, N. J., NanoSchiavi, M. L., Baier, M. L., Frohman, E. M., Winslow, H., Frohman, T. C., Calabresi, P. A., Maguire, M. G., Cutter, G. R., and Balcer, L. J. (2006). Relation of visual function to retinal nerve fiber layer thickness in multiple sclerosis. Ophthalmology 113, 324-332.

Fitzgerald, D. C., Ciric, B., Touil, T., Harle, H., Grammatikopolou, J., Das Sarma, J., Gran, B., Zhang, G. X., and Rostami, A. (2007). Suppressive effect of IL-27 on

with the higher dose of resveratrol. This difference may be due to higher concentration of MOG used to induce a more robust EAE disease course in the current studies. While Singh's studies (Singh et al., 2007) reported some decrease in spinal cord inflammation, the level of inflammation was not quantified and the sections they showed demonstrated that obvious foci of inflammation still occurred in EAE spinal cords after resveratrol treatment, similar to our findings. Singh did demonstrate some changes in serum levels of cytokines 25 days post-immunization, although the IL-17 cytokine felt to be critical in EAE was not significantly affected by resveratrol treatment at day 25 despite earlier effects at day 9 (Singh et al., 2007). Those studies did not evaluate inflammatory cell types or cytokine profiles in the CNS, the site of damage in EAE. Our current results therefore examined this, and found no significant effects of resveratrol. Together, results do suggest resveratrol may have some modest immunomodulatory effects at some points in the course of autoimmune demyelinating disease, but its neuroprotective effects are most prominent.

Another study demonstrated some immunomodulatory effects, altering the percentage if IL-17 positive T cells in the periphery and CNS following long-term resveratrol treatment in the relapsing-remitting EAE model (Imler and Petro, 2009). However, like our prior studies, they also saw little effect during the first acute episode in the relapsing-remitting disease, only noting some suppression after multiple relapses. Resveratrol may therefore have some long-term immunomodulatory effects itself that could have additional benefits in EAE and MS. Future studies in both EAE models using combination therapy with resveratrol and current immunomodulatory MS medications will be useful to determine whether resveratrol can enhance their immunosuppressive effects, and retain its own important neuroprotective effects.

\section{ACKNOWLEDGMENTS}

The authors thank Sirtris, a GSK Company, for kindly providing the SRT501 compound for these studies. This work was supported by National Institutes of Health grants EY015098 and EY019014, grant RG 4214-A-1 from the National Multiple Sclerosis Society, Research to Prevent Blindness, and the F. M. Kirby Foundation.

encephalitogenic Th17 cells and the effector phase of experimental autoimmune encephalomyelitis. J. Immunol. 179, 3268-3275.

Gran, B., Zhang, G. X., and Rostami, A. (2004). Role of the IL-12/IL-23 system in the regulation of Tcell responses in central nervous system inflammatory demyelination. Crit. Rev. Immunol. 24, 111-128.

Hickman, S. J., Kapoor, R., Jones, S. J., Altmann, D. R., Plant, G. T., and Miller, D. H. (2003). Corticosteroids do not prevent optic nerve atrophy following optic neuritis. J. Neurol. Neurosurg. Psychiatr. 74, 1139-1141.
Hussain, R. Z., Hopkins, S. C., Frohman, E. M., Eagar, T. N., Cravens, P. C., Greenberg, B. M., Vernino, S., and Stüve, O. (2009). Direct and consensual murine pupillary reflex metrics: establishing normative values. Auton. Neurosci. 151, 164-167.

Imler, T. J. Jr., and Petro, T. M. (2009) Decreased severity of experimental autoimmune encephalomyelitis during resveratrol administration is associated with increased IL-17+IL10+ T cells, CD4(-) IFN-gamma+ cells, and decreased macrophage IL6 expression. Int. Immunopharmacol. 9, 134-143. 
Jacobs, L. D., Cookfair, D. L., Rudick, R. A., Herndon, R. M., Richert, J. R., Salazar, A. M., Fischer, J. S., Goodkin, D. E., Granger, C. V., Simon, J. H., Alam, J. J., Bartoszak, D. M., Bourdette, D. M., Braiman, J., Brownscheidle, C. M., Coats, M. E., Cohan, S. L., Dougherty, D. S., Kinkel, R. P., Mass, M. K., Munschauer, F. E. III, Priore, R. L., Pullicino, P. M., Scherokman, B. J., WeinstockGuttman, B., Whitham, R. H., and The Multiple Sclerosis Collaborative Research Group. (1996). Intramuscular interferon beta-1a for disease progression in relapsing multiple sclerosis. Ann. Neurol. 39, 285-294.

Johnson, K. P., Brooks, B. R., Cohen, J. A., Ford, C. C., Goldstein, J., Lisak, R. P., Myers, L. W., Panitch, H. S., Rose, J. W., Schiffer, R. B., Vollmer, T., Weiner, L. P., Wolinsky, J. S., and the Copolymer 1 Multiple Sclerosis Study Group. (1995). Copolymer 1 reduces relapse rate and improves disability in relapsingremitting multiple sclerosis: results of a phase III multicenter, doubleblind, placebo-controlled trial. Neurology 45, 1268-1276.

Langrish, C. L., Chen, Y., Blumenschein, W. M., Mattson, J., Basham, B., Sedgwick, J. D., McClanahan, T., Kastelein, R. A., and Cua, D. J. (2005). IL-23 drives a pathogenic T cell population that induces autoimmune inflammation. J. Exp. Med. 201, 233-240.

Lock, C., Hermans, G., Pedotti, R., Brendolan, A., Schadt, E., Garren, H., Langer-Gould, A., Strober, S., Cannella, B., Allard, J., Klonowski, P., Austin, A., Lad, N., Kaminski, N., Galli, S. J., Oksenberg, J. R., Raine, C. S., Heller, R., and Steinman, L. (2002). Gene microarray analysis of multiple sclerosis lesions yields new targets validated in autoimmune encephalomyelitis. Nat. Med. 8, 500-508.

Losseff, N. A., Webb, S. L., O'Riordan, J. I., Page, R., Wang, L., Barker, G. J., Tofts, P. S., McDonald, W. I., Miller, D. H., and Thompson, A. J. (1996a). Spinal cord atrophy and disability in multiple sclerosis: a new reproducible and sensitive MRI method with potential to monitor disease progression. Brain 119, 701-708.

Losseff, N. A., Wang, L., Lai, H. M., Yoo, D. S., Gawne-Cain, M. L., McDonald, W. I., Miller, D. H., and Thompson, A. J. (1996b). Progressive cerebral atrophy in multiple sclerosis: a serial MRI study. Brain 119, 2009-2019.

Milne, J. C., Lambert, P. D., Schenk, S., Carney, D. P., Smith, J. J., Gagne, D. J., Jin, L., Boss, O., Perni, R. B., Vu, C. B., Bemis, J. E., Xie, R., Disch, J. S., Ng, P. Y., Nunes, J. J., Lynch, A. V., Yang, H., Galonek, H., Israelian, K., Choy, W., Iffland, A., Lavu, S., Medvedik, O., Sinclair, D. A., Olefsky, J. M., Jirousek, M. R., Elliott, P. J., and Westphal, C. H. (2007). Small molecule activators of SIRT 1 as therapeutics for the treatment of type 2 diabetes. Nature 450, 712-716.

Noseworthy, J. H., Lucchinetti, C., Rodriguez, M., and Weinshenker, B. G. (2000). Multiple sclerosis. $N$. Engl. J. Med. 343, 938-952.

Parisi, V., Manni, G., Spadaro, M., Colacino, G., Restuccia, R., Marchi, S., Bucci, M. G., and Pierelli, F. (1999). Correlation between morphological and functional retinal impairment in multiple sclerosis patients. Invest. Ophthalmol. Vis. Sci. 40, 2520-2527.

Parry, A., Corkill, R., Blamire, A. M., Palace, J., Narayanan, S., Arnold, D., Styles, P., and Matthews, P. M. (2003). Beta-Interferon treatment does not always slow the progression of axonal injury in multiple sclerosis. J. Neurol. 250, 171-178.

Prusky, G. T., Alam, N. M., Beekman, S., and Douglas, R. M. (2004). Rapid quantification of adult and developing mouse spatial vision using a virtual optomotor system. Invest. Ophthalmol. Vis. Sci. 45, 4611-4616.

Quinn, T., Dutt, M., and Shindler, K. S. (2011). Optic neuritis and retinal ganglion cell loss in a chronic murine model of multiple sclerosis. Front. Neurol. 2:50. doi:10.3389/fneur.2011.00050

Shao, H., Huang, Z., Sun, S. L., Kaplan, H. J., and Sun, D.
(2004). Myelin/oligodendrocyte glycoprotein-specific T-cells induce severe optic neuritis in the C57BL/6 mouse. Invest. Ophthalmol. Vis. Sci. 45, 4060-4065.

Shindler, K. S., Chatterjee, D., Biswas, K., Goyal, A., Dutt, M., Nassrallah, M., Khan, R. S., and Das Sarma, J. (2011). Macrophage-mediated optic neuritis induced by retrograde axonal transport of spike gene recombinant mouse hepatitis virus. J. Neuropathol. Exp. Neurol. 70, 470-480.

Shindler, K. S., Guan, Y., Ventura, E. Bennett, J., and Rostami, A. (2006). Retinal ganglion cell loss induced by acute optic neuritis in a relapsing model of multiple sclerosis. Mult. Scler. 12, 526-532.

Shindler, K. S., Revere, K., Dutt, M., Ying, G.-S., and Chung, D. C. (2012). In vivo detection of experimental optic neuritis by pupillometry. Exp. Eye Res. doi: 10.1016/j.exer.2012.04.005

Shindler, K. S., Ventura, E., Dutt, M., Elliott, P., Fitzgerald, D. C., and Rostami, A. (2010). Oral resveratrol reduces neuronal damage in a model of multiple sclerosis. J. Neuroophthalmol. 30, 328-339.

Shindler, K. S., Ventura, E., Rex, T. S. Elliott, P., and Rostami, A. (2007). SIRT1 activation confers neuroprotection in experimental optic neuritis. Invest. Ophthalmol. Vis. Sci. 48, 3602-3609.

Singh, N. P., Hegde, V. L., Hofseth, L. J., Nagarkatti, M., and Nagarkatti, P. (2007). Resveratrol (trans-3,5,4'-trihydroxystilbene) ameliorates experimental allergic encephalomyelitis, primarily via induction of apoptosis in $\mathrm{T}$ cells involving activation of aryl hydrocarbon receptor and estrogen receptor. Mol. Pharmacol. 72, 1508-1521.

Steel, D. H. W., and Waldock, A. (1998). Measurement of the retinal nerve fiber layer with scanning laser polarimetry in patients with previous demyelinating optic neuritis. J. Neurol. Neurosurg. Psychiatr. 64, 505-509.
Steinman, L. (2008). A rush to judgment on Th17. J. Exp. Med. 205 1517-1522.

Stromnes, I. M., Cerretti, L. M., Liggitt, D., Harris, R. A., and Goverman, J. M. (2008). Differential regulation of central nervous system autoimmunity by $\mathrm{T}(\mathrm{H}) 1$ and $\mathrm{T}(\mathrm{H}) 17$ cells. Nat. Med. 14, 337-342.

Trapp, B. D., Peterson, J., Ransohoff, R. M., Rudick, R., Mork, S., and Bo, L. (1998). Axonal transection in the lesions of multiple sclerosis. N. Engl. J. Med. 338, 278-285.

Trip, S. A., Schlottmann, P. G., Jones, S. J., Altmann, D. R., Garway-Heath, D. F., Thompson, A. J., Plant, G. T., and Miller, D. H. (2005). Retinal nerve fiber layer axonal loss and visual dysfunction in optic neuritis. Ann. Neurol. 58, 383-391.

Weiner, H. L., Hafler, D. A., Fallis, R. J., Johnson, D., Ault, K. A., and Hauser, S. L. (1984). T cell subsets in patients with multiple sclerosis. An overview. Ann. N. Y. Acad. Sci. 436, 281-293.

Conflict of Interest Statement: The authors declare that the research was conducted in the absence of any commercial or financial relationships that could be construed as a potential conflict of interest.

Received: 19 March 2012; accepted: 28 April 2012; published online: 24 May 2012.

Citation: Fonseca-Kelly Z, Nassrallah $M$, Uribe J, Khan RS, Dine K, Dutt $M$ and Shindler KS (2012) Resveratrol neuroprotection in a chronic mouse model of multiple sclerosis. Front. Neur. 3:84. doi: 10.3389/fneur.2012.00084

This article was submitted to Frontiers in Neuro-Ophthalmology, a specialty of Frontiers in Neurology.

Copyright (c) 2012 Fonseca-Kelly, Nassrallah, Uribe, Khan, Dine, Dutt and Shindler. This is an open-access article distributed under the terms of the Creative Commons Attribution Non Commercial License, which permits noncommercial use, distribution, and reproduction in other forums, provided the original authors and source are credited. 